\title{
CLOAKING USING COMPLEMENTARY MEDIA FOR THE HELMHOLTZ EQUATION AND A THREE SPHERES INEQUALITY FOR SECOND ORDER ELLIPTIC EQUATIONS
}

\author{
HOAI-MINH NGUYEN AND LOC HOANG NGUYEN
}

\begin{abstract}
Cloaking using complementary media was suggested by Lai et al. in 2009. This was proved by H.-M. Nguyen (2015) in the quasistatic regime. One of the difficulties in the study of this problem is the appearance of the localized resonance, i.e., the fields blow up in some regions and remain bounded in some others as the loss goes to 0 . To this end, H.-M. Nguyen introduced the technique of removing localized singularity and used a standard three spheres inequality. The method used also works for the Helmholtz equation. However, it requires small size of the cloaked region for large frequency due to the use of the (standard) three spheres inequality. In this paper, we give a proof of cloaking using complementary media in the finite frequency regime without imposing any condition on the cloaked region; the cloak works for an arbitrary fixed frequency provided that the loss is sufficiently small. To successfully apply the above approach of Nguyen, we establish a new three spheres inequality. A modification of the cloaking setting to obtain illusion optics is also discussed.
\end{abstract}

\section{INTRODUCTION}

Negative index materials (NIMs) were investigated theoretically by Veselago in [36]. The existence of such materials was confirmed by Shelby, Smith, and Schultz in [35]. The study of NIMs has attracted a lot of attention in the scientific community thanks to their interesting properties and applications. An appealing one is cloaking using complementary media.

Cloaking using NIMs or more precisely cloaking using complementary media was suggested by Lai et al. in [11]. Their work was inspired by the notion of complementary media suggested by Pendry and Ramakrishna in [32. Cloaking using complementary media was established in [21] in the quasistatic regime using slightly different schemes from [11. Two difficulties in the study of cloaking using complementary media are as follows. Firstly, this problem is unstable since the equations describing the phenomenon have sign changing coefficients, hence the ellipticity and the compactness are lost. Secondly, the localized resonance, i.e., the field blows up in some regions and remains bounded in some others, might appear. To handle these difficulties, in 21] the author introduced the removing localized

Received by the editors March 10, 2015 and, in revised form, August 27, 2015.

2010 Mathematics Subject Classification. Primary 35B34, 35B35, 35B40, 35J05, 78A25, $78 \mathrm{M} 35$.

Key words and phrases. Cloaking, illusion optics, superlensing, three spheres inequality, localized resonance, negative index materials, complementary media.

This research was partially supported by NSF grant DMS-1201370 and by the Alfred P. Sloan Foundation. 
singularity technique and used a standard three spheres inequality. The approach in 21] also involved the reflecting technique introduced in [18. The method in 21] also works for the Helmholtz equation; however since the largest radius in the (standard) three spheres inequality is small as frequency is large (see Section 2 for further discussion), the size of the cloaked region is required to be small for large frequency.

In this paper, we present a proof of cloaking using complementary media in the finite frequency regime. Our goal is to not impose any condition on the size of the cloaked region (Theorem 1); the cloak works for an arbitrary fixed frequency as long as the loss is sufficiently small. To successfully apply the approach in [21, we establish a new three spheres inequality for the second order elliptic equations which holds for arbitrary radius (Theorem 2 in Section 2). This inequality is inspired from the unique continuation principle and its proof is in the spirit of Protter in 34. A modification of the cloaking setting to obtain illusion optics is discussed in Section 4 (Theorem 3). This involves the idea of superlensing in [19]. Cloaking using complementary media for electromagnetic waves is investigated in 24 .

In addition to cloaking using complementary media, other application of NIMs are superlensing using complementary media as suggested in [29, 30, 33] (see also [28]) and confirmed in [19,22, and cloaking via anomalous localized resonance [15] (see also [3, 10, 20]). Complementary media were studied in a general setting in [18,22] and played an important role in these applications; see [17, 19, 22, 25].

Let us describe the problem more precisely. Assume that the cloaked region is the annulus $B_{\gamma r_{2}} \backslash B_{r_{2}}$ for some $r_{2}>0$ and $1<\gamma<2$ in which the medium is characterized by a matrix $a$ and a function $\sigma$. The assumption on the cloaked region by all means imposes no restriction since any bounded set is a subset of such a region provided that the radius and the origin are appropriately chosen. The idea suggested by Lai et al. in [11] in two dimensions is to construct its complementary medium in $B_{r_{2}} \backslash B_{r_{1}}$ for some $0<r_{1}<r_{2}$.

In this paper, instead of taking the schemes of Lai et al., we use a scheme from 21] which is inspired but different from the ones from [11]. Following [21, the cloak contains two parts. The first one, in $B_{r_{2}} \backslash B_{r_{1}}$, makes use of complementary media to cancel the effect of the cloaked region, and the second one, in $B_{r_{1}}$, is to fill the space which "disappears" from the cancellation by the homogeneous media. Concerning the first part, instead of $B_{\gamma r_{2}} \backslash B_{r_{2}}$, we consider $B_{r_{3}} \backslash B_{r_{2}}$ with $r_{3}=2 r_{2}$ (the constant 2 considered here is just a matter of simple representation) as the cloaked region in which the medium is given by

$$
\hat{a}, \hat{\sigma}=\left\{\begin{array}{cl}
a, \sigma & \text { in } B_{\gamma r_{2}} \backslash B_{r_{2}}, \\
I, 1 & \text { in } B_{r_{3}} \backslash B_{\gamma r_{2}} .
\end{array}\right.
$$

The complementary medium in $B_{r_{2}} \backslash B_{r_{1}}$ is given by

$$
-F_{*}^{-1} \hat{a},-F_{*}^{-1} \hat{\sigma},
$$

where $F: B_{r_{2}} \backslash \bar{B}_{r_{1}} \rightarrow B_{r_{3}} \backslash \bar{B}_{r_{2}}$ is the Kelvin transform with respect to $\partial B_{r_{2}}$, i.e.,

$$
F(x)=\frac{r_{2}^{2}}{|x|^{2}} x .
$$

Here

$$
T_{*} \hat{a}(y)=\frac{D T(x) \hat{a}(x) D T(x)^{T}}{J(x)} \quad \text { and } \quad T_{*} \hat{\sigma}(y)=\frac{\hat{\sigma}(x)}{J(x)}
$$


where $x=T^{-1}(y)$ and $J(x)=|\operatorname{det} D T(x)|$ for a diffeomorphism $T$. It follows that

$$
r_{1}=r_{2}^{2} / r_{3}
$$

Concerning the second part, the medium in $B_{r_{1}}$ is given by

$$
\left(r_{3}^{2} / r_{2}^{2}\right)^{d-2} I,\left(r_{3}^{2} / r_{2}^{2}\right)^{d}
$$

The reason for this choice will be explained later.

With the loss, the medium is characterized by $s_{\delta} A, s_{0} \Sigma(\delta>0)$, where

$$
A, \Sigma=\left\{\begin{array}{cl}
\hat{a}, \hat{\sigma} & \text { in } B_{r_{3}} \backslash B_{r_{2}}, \\
F_{*}^{-1} \hat{a}, F_{*}^{-1} \hat{\sigma} & \text { in } B_{r_{2}} \backslash B_{r_{1}}, \\
\left(r_{3}^{2} / r_{2}^{2}\right)^{d-2} I,\left(r_{3}^{2} / r_{2}^{2}\right)^{d} & \text { in } B_{r_{1}}, \\
I, 1 & \text { otherwise }
\end{array}\right.
$$

and, for $\delta \geq 0$,

$$
s_{\delta}:=\left\{\begin{array}{cl}
-1+i \delta & \text { in } B_{r_{2}} \backslash B_{r_{1}}, \\
1 & \text { otherwise. }
\end{array}\right.
$$

Physically, the imaginary part of $s_{\delta} A$ is the loss of the medium (more precisely the loss of the medium in $B_{r_{2}} \backslash B_{r_{1}}$ ). Here and in what follows, we assume that

$$
\frac{1}{\Lambda}|\xi|^{2} \leq a(x) \xi \cdot \xi \leq \Lambda|\xi|^{2} \quad \forall \xi \in \mathbb{R}^{d} \text {, for a.e. } x \in B_{\gamma r_{2}} \backslash B_{r_{2}},
$$

for some $\Lambda \geq 1$. In what follows, we assume in addition that

$$
\hat{a} \text { is Lipschitz in } B_{r_{3}} \backslash B_{r_{2}} \text {. }
$$

One can verify that medium $\left(s_{0} A, s_{0} \Sigma\right)$ is of reflecting complementary property, a concept introduced in [18, Definition 1], by considering the diffeomorphism $G$ : $\mathbb{R}^{d} \backslash \bar{B}_{r_{3}} \rightarrow B_{r_{3}} \backslash\{0\}$ which is the Kelvin transform with respect to $\partial B_{r_{3}}$, i.e.,

$$
G(x)=r_{3}^{2} x /|x|^{2} .
$$

It is important to note that

$$
G_{*} F_{*} A=I \text { and } G_{*} F_{*} 1=1 \text { in } B_{r_{3}},
$$

since $G \circ F(x)=\left(r_{3}^{2} / r_{2}^{2}\right) x$. This is the reason for choosing $(A, \Sigma)$ in (1.3).

Let $\Omega$ be a smooth open subset of $\mathbb{R}^{d}(d=2,3)$ such that $B_{r_{3}} \subset \subset \Omega$. Given $f \in L^{2}(\Omega)$, let $u_{\delta}, u \in H_{0}^{1}(\Omega)$ be respectively the unique solution to

$$
\operatorname{div}\left(s_{\delta} A \nabla u_{\delta}\right)+s_{0} k^{2} \Sigma u_{\delta}=f \text { in } \Omega,
$$

and

$$
\Delta u+k^{2} u=f \text { in } \Omega
$$

As in [18, we assume that

$$
\text { equation (1.11) with } f=0 \text { has only a zero solution in } H_{0}^{1}(\Omega) \text {. }
$$


Our result on cloaking using complementary media is:

Theorem 1. Let $d=2,3, f \in L^{2}(\Omega)$ with $\operatorname{supp} f \subset \Omega \backslash B_{r_{3}}$ and let $u$ and $u_{\delta}$ in $H_{0}^{1}(\Omega)$ be the unique solution to (1.10) and (1.11), resp. There exists $\gamma_{0}>1$, depending only on $\Lambda$ and the Lipschitz constant of $\hat{a}$, such that if $1<\gamma<\gamma_{0}$, then

$$
u_{\delta} \rightarrow u \text { weakly in } H^{1}\left(\Omega \backslash B_{r_{3}}\right) \text { as } \delta \rightarrow 0 .
$$

For an observer outside $B_{r_{3}}$, the medium in $B_{r_{3}}$ looks like the homogeneous one by (1.13) (and also (1.11)): one has cloaking.

Remark 1. Since $\Delta\left(u_{\delta}-u\right)+k^{2}\left(u_{\delta}-u\right)=0$ in $\Omega \backslash B_{r_{3}}$, it follows from Theorem 1 that $u_{\delta} \rightarrow u$ in $C_{\text {loc }}^{m}\left(\Omega \backslash \bar{B}_{r_{3}}\right)$ for $m \in \mathbb{N}$. A discussion on the rate of the convergence is given in Remark 4 after the proof of Theorem 1 .

Remark 2. The constant $\gamma_{0}$ in Theorem 1 depends only on $\Lambda$ and the Lipschitz constant of $\hat{a}$. Hence, by choosing $r_{2}$ large enough and $\gamma=\gamma_{0} / 2$, the cloaked region $B_{\gamma r_{2}} \backslash B_{r_{2}}$ can be arbitrarily large.

Remark 3. The case $k=0$ was established in [21]. The proof of Theorem 1 has its root from there.

The proof of Theorem 1 is given in Section 3, It is based on the removing localized singularity technique introduced in 21] and uses a new three sphere inequality (Theorem 2) discussed in the next section. The discussion on illusion optics is given in Section 4 .

\section{Three SPHERES INEQUALITIES}

Let $v$ be a holomorphic function defined in $B_{R_{3}}$. Hadamard in [8] proved the following famous three spheres inequality:

$$
\|v\|_{L^{\infty}\left(\partial B_{R_{2}}\right)} \leq\|v\|_{L^{\infty}\left(\partial B_{R_{1}}\right)}^{\alpha}\|v\|_{L^{\infty}\left(\partial B_{r_{3}}\right)}^{1-\alpha},
$$

for all $0<R_{1}<R_{2}<R_{3}$, where

$$
\alpha=\log \left(\frac{R_{3}}{R_{2}}\right) / \log \left(\frac{R_{3}}{R_{1}}\right) .
$$

A three spheres inequality for general elliptic equations was proved by Landis 13 using Carleman type estimates. Landis proved [13, Theorem 2.1] that 1 if $v$ is a solution to

$$
\operatorname{div}(M \nabla v)+\vec{b} \cdot \nabla v+c v=0 \text { in } B_{R_{3}},
$$

where $M$ is elliptic, symmetric, and of class $C^{2}, \vec{b}, c \in C^{1}$, and $c \leq 0$, then there is a constant $C>0$ such that

$$
\|v\|_{L^{\infty}\left(\partial B_{R_{2}}\right)} \leq C\|v\|_{L^{\infty}\left(\partial B_{R_{1}}\right)}^{\alpha}\|v\|_{L^{\infty}\left(\partial B_{r_{3}}\right)}^{1-\alpha},
$$

for some $\alpha \in(0,1)$ depending only on $R_{2} / R_{1}, R_{2} / R_{3}$, the ellipticity constant of $M$, and the regularity constants of $M, \vec{b}$, and $c$. The assumption $c \leq 0$ is crucial and this is discussed in the next paragraph. Another proof was obtained by Agmon [1] in which he used the logarithmic convexity. Garofalo and Lin in [6] established

\footnotetext{
${ }^{1}$ In fact, [13 Theorem 2.1] deals with the non-divergent form; however since $M$ is assumed $C^{2}$, the two forms are equivalent.
} 
similar results where the $L^{\infty}$-norm is replaced by the $L^{2}$-norm, $M$ is of class $C^{1}$, and $\vec{b}$ and $c$ are in $L^{\infty}$ :

$$
\|v\|_{L^{2}\left(\partial B_{R_{2}}\right)} \leq C\|v\|_{L^{2}\left(\partial B_{R_{1}}\right)}^{\alpha}\|v\|_{L^{2}\left(\partial B_{r_{3}}\right)}^{1-\alpha}
$$

using the frequency function.

A typical example of (2.2) when $c>0$ is the Helmholtz equation:

$$
\Delta v+k^{2} v=0 \text { in } B_{R_{3}} .
$$

Given $k>0$, neither (2.4) nor (2.3) holds for all $R_{1}<R_{2}<R_{3}$. Indeed, consider first the case $d=2$. It is clear that for $n \in \mathbb{Z} \backslash\{0\}$, the function $J_{n}(k r) e^{i n \theta}$ is a solution to (2.5) in $\mathbb{R}^{2} \backslash\{0\}$, where $J_{n}$ is the Bessel function of order $n$. By taking $R_{1}, R_{2}$, and $R_{3}$ such that $J_{n}\left(k R_{1}\right)=0 \neq J_{n}\left(k R_{2}\right)$, one reaches the fact that neither (2.4) nor (2.3) is valid. The same conclusion holds in the higher dimensional case by similar arguments. In the case $c>0$, (2.4) holds under the smallness of $R_{3}$ (see, e.g., [2, Theorem 4.1]); this condition is equivalent to the smallness of $c$ for a fixed $R_{3}$ by a scaling argument.

In this paper, we establish a new type of three spheres inequalities without imposing the smallness condition on $R_{3}$. This inequality will play an important role in the proof of Theorem 1 Define

$$
\|v\|_{\mathbf{H}\left(\partial B_{r}\right)}=\|v\|_{H^{1 / 2}\left(\partial B_{r}\right)}+\|M \nabla v \cdot \nu\|_{H^{-1 / 2}\left(\partial B_{r}\right)} .
$$

Here and in what follows, $\nu$ denotes the outward normal vector on a sphere.

Our result on three spheres inequalities is:

Theorem 2. Let $d \geq 2, c_{1}, c_{2}>0,0<R_{*}<R_{1}<R_{2}<R_{3}<R^{*}$, and let $M$ be a Lipschitz uniformly elliptic symmetric matrix-valued function defined in $B_{R^{*}}$. Assume $v \in H^{1}\left(B_{R_{3}} \backslash \bar{B}_{R_{1}}\right)$ satisfies

$$
|\operatorname{div}(M \nabla v)| \leq c_{1}|\nabla v|+c_{2}|v|, \quad \text { in } B_{R_{3}} \backslash \bar{B}_{R_{1}} .
$$

There exists a constant $q \geq 1$, depending only on $d$ and the elliptic and the Lipschitz constants of $M$, such that, for any $\lambda_{0}>1$ and $R_{2} \in\left(\lambda_{0} R_{1}, R_{3} / \lambda_{0}\right)$, we have

$$
\|v\|_{\mathbf{H}\left(\partial B_{R_{2}}\right)} \leq C\|v\|_{\mathbf{H}\left(\partial B_{R_{1}}\right)}^{\alpha}\|v\|_{\mathbf{H}\left(\partial B_{R_{3}}\right)}^{1-\alpha}, \quad \text { where } \quad \alpha:=\frac{R_{2}^{-q}-R_{3}^{-q}}{R_{1}^{-q}-R_{3}^{-q}}
$$

Here $C$ is a positive constant depending on the elliptic and the Lipschitz constants of $M, c_{1}, c_{2}, R_{*}, R^{*}, d$, and $\lambda_{0}$ but independent of $v$.

In Theorem 2, one does not impose any smallness condition on $R_{1}, R_{2}, R_{3}$ and the exponent $\alpha$ is independent of $c_{1}$ and $c_{2}$. The proof of Theorem 2 is inspired by the approach of Protter in [34]. Nevertheless, different test functions are used. The ones in [34] are too concentrated at 0 and not suitable for our purpose. The connection between three spheres inequalities and the unique continuation principle, and the application of three spheres inequalities for the stability of Cauchy problems can be found in [2].

The rest of this section contains two subsections. In the first one, we present some lemmas used in the proof of Theorem 2, The proof of Theorem 2 is given in the second subsection. 
2.1. Preliminaries. This section contains several lemmas used in the proof of Theorem 2. These lemmas are in the spirit of [34. Nevertheless, the test functions used here are different from there. Let $0<R_{1}<R_{3}<+\infty$. In this section, we assume that $M$ is a Lipschitz symmetric matrix-valued function defined in $\bar{B}_{R_{3}} \backslash B_{R_{1}}$ and satisfies

$$
\frac{1}{\Lambda}|\xi|^{2} \leq M(x) \xi \cdot \xi \leq \Lambda|\xi|^{2} \quad \forall \xi \in \mathbb{R}^{d},
$$

for a.e. $x \in \bar{B}_{R_{3}} \backslash B_{R_{1}}$, for some $\Lambda \geq 1$. Set

$$
L:=\|M\|_{L^{\infty}}+R_{3}\|\nabla M\|_{L^{\infty}} .
$$

All functions considered in this section are assumed to be real.

The first lemma is:

Lemma 1. Let $d \geq 2$ and $z \in H^{2}\left(B_{R_{3}} \backslash \bar{B}_{R_{1}}\right)$. We have

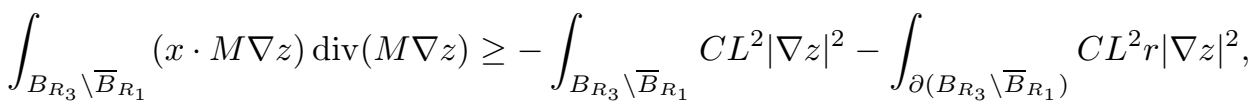

for some positive constant $C$ depending only on $d$.

Proof. An integration by parts gives

$$
\begin{aligned}
\int_{B_{R_{3}} \backslash \bar{B}_{R_{1}}}(x \cdot M \nabla z) \operatorname{div}(M \nabla z)=- & \int_{B_{R_{3}} \backslash \bar{B}_{R_{1}}} \nabla(x \cdot M \nabla z) \cdot M \nabla z \\
& +\int_{\partial\left(B_{\left.R_{3} \backslash \bar{B}_{R_{1}}\right)}(x \cdot M \nabla z) M \nabla z \cdot \nu .\right.}
\end{aligned}
$$

Using the symmetry of $M$, we have2

$$
\begin{aligned}
\frac{\partial}{\partial x_{i}}(x \cdot M \nabla z) & =\frac{\partial}{\partial x_{i}}\left(M_{k j} x_{j} \frac{\partial z}{\partial x_{k}}\right) \\
& =M_{k j} x_{j} \frac{\partial^{2} z}{\partial x_{i} \partial x_{k}}+M_{k i} \frac{\partial z}{\partial x_{k}}+x_{j} \frac{\partial M_{k j}}{\partial x_{i}} \frac{\partial z}{\partial x_{k}}
\end{aligned}
$$

and

$$
\begin{array}{r}
-\int_{B_{R_{3}} \backslash \bar{B}_{R_{1}}} 2 x_{j} M_{k j} \frac{\partial^{2} z}{\partial x_{i} \partial x_{k}} M_{i l} \frac{\partial z}{\partial x_{l}}=-\int_{B_{R_{3}} \backslash \bar{B}_{R_{1}}} x_{j} M_{k j} M_{i l} \frac{\partial}{\partial x_{k}}\left(\frac{\partial z}{\partial x_{i}} \frac{\partial z}{\partial x_{l}}\right) \\
=\int_{B_{R_{3}} \backslash \bar{B}_{R_{1}}} \frac{\partial\left(x_{j} M_{k j} M_{i l}\right)}{\partial x_{k}} \frac{\partial z}{\partial x_{i}} \frac{\partial z}{\partial x_{l}}-\int_{\partial\left(B_{R_{3}} \backslash \bar{B}_{R_{1}}\right)} x_{j} M_{k j} M_{i l} \frac{\partial z}{\partial x_{i}} \frac{\partial z}{\partial x_{l}} \nu_{k} .
\end{array}
$$

We derive from (2.11) and (2.12) that

$-\int_{B_{R_{3}} \backslash \bar{B}_{R_{1}}} \nabla(x \cdot M \nabla z) \cdot M \nabla z \geq-\int_{B_{R_{3}} \backslash \bar{B}_{R_{1}}} C L^{2}|\nabla z|^{2}-\int_{\partial\left(B_{R_{3}} \backslash \bar{B}_{R_{1}}\right)} C L^{2} r|\nabla z|^{2}$.

The conclusion now follows from (2.10) and (2.13).

\footnotetext{
${ }^{2}$ In what follows, the repeated summation is used.
} 
The second lemma is

Lemma 2. Let $d \geq 2, \beta \in \mathbb{R}$, and $z \in H^{2}\left(B_{R_{3}} \backslash \bar{B}_{R_{1}}\right)$. There exists $p_{\Lambda, L} \geq 1$ such that if $p \geq p_{\Lambda, L}$ and $|\beta| R_{3}^{-p} \geq 2$, then

$$
\begin{aligned}
& \int_{B_{R_{3}} \backslash \bar{B}_{R_{1}}} e^{\beta r^{-p}}(M x\left.\cdot \nabla|z|^{2}\right) \operatorname{div}\left(M \nabla e^{-\beta r^{-p}}\right)+\int_{\partial\left(B_{R_{3}} \backslash \bar{B}_{R_{1}}\right)} C L^{2} p^{2} \beta^{2} r^{-2 p-1}|z|^{2} \\
& \geq \int_{B_{R_{3}} \backslash \bar{B}_{R_{1}}} \frac{1}{2} \Lambda^{-2} p^{3} \beta^{2} r^{-2 p-2}|z|^{2}-\int_{B_{R_{3}} \backslash \bar{B}_{R_{1}}} C L^{2}|\nabla z|^{2}
\end{aligned}
$$

for some positive constant $C$ depending only on $d$.

Proof. A computation yields

$$
\begin{aligned}
\operatorname{div}\left(M \nabla e^{-\beta r^{-p}}\right)= & p \beta e^{-\beta r^{-p}}\left[p \beta r^{-2 p-4}-(p+2) r^{-p-4}\right] x \\
& \cdot M x+p \beta r^{-p-2} e^{-\beta r^{-p}} \operatorname{div}(M x) .
\end{aligned}
$$

An integration by parts gives

$$
\int_{B_{R_{3}} \backslash \bar{B}_{R_{1}}} e^{\beta r^{-p}}\left(M x \cdot \nabla|z|^{2}\right) \operatorname{div}\left(M \nabla e^{-\beta r^{-p}}\right)=P+Q .
$$

Here

$$
P=P_{1}+P_{2}+P_{3}
$$

with

$$
\left\{\begin{array}{l}
P_{1}=-\int_{B_{R_{3}} \backslash \bar{B}_{R_{1}}} p^{2} \beta^{2}|z|^{2} \operatorname{div}\left[r^{-2 p-4}(x \cdot M x) M x\right] \\
P_{2}=\int_{B_{R_{3}} \backslash \bar{B}_{R_{1}}} p \beta(p+2)|z|^{2} \operatorname{div}\left[r^{-p-4}(x \cdot M x) M x\right] \\
P_{3}=\int_{B_{R_{3}} \backslash \bar{B}_{R_{1}}} 2 p \beta r^{-p-2} \operatorname{div}(M x) z \nabla z \cdot M x
\end{array}\right.
$$

and

$$
Q=\int_{\partial\left(B_{R_{3}} \backslash \bar{B}_{R_{1}}\right)} p \beta|z|^{2}\left(\left[p \beta r^{-2 p-4}-(p+2) r^{-p-4}\right] x \cdot M x\right) M x \cdot \nu .
$$

We next estimate $P$ and $Q$. A computation yields

$$
-\operatorname{div}\left[r^{-2 p-4}(x \cdot M x) M x\right]=(2 p+4)(x \cdot M x)^{2} r^{-2 p-6}-r^{-2 p-4} \operatorname{div}[(x \cdot M x) M x] .
$$

This implies

$$
P_{1} \geq \int_{B_{R_{3}} \backslash \bar{B}_{R_{1}}} p^{2} \beta^{2} r^{-2 p-2}|z|^{2}\left[(2 p+4) \Lambda^{-2}-C L^{2}\right] .
$$

Similarly,

$$
P_{2} \geq-\int_{B_{R_{3}} \backslash \bar{B}_{R_{1}}}(p+2) p|\beta| r^{-p-2}|z|^{2}\left[(p+4) \Lambda^{-2}+C L^{2}\right] .
$$

A combination of (2.15) and (2.16) yields

$$
P_{1}+P_{2} \geq \int_{B_{R_{3}} \backslash \bar{B}_{R_{1}}} \Lambda^{-2} p^{3} \beta^{2} r^{-2 p-2}|z|^{2} .
$$


Here we used the fact that $p \geq p_{\Lambda, L}$ and $|\beta| R_{3}^{-p} \geq 2$. On the other hand, using Cauchy's inequality, we have

$$
\left|P_{3}\right| \leq \int_{B_{R_{3}} \backslash \bar{B}_{R_{1}}} p^{2} \beta^{2} r^{-2 p-2} L^{2}|z|^{2}+\int_{B_{R_{3}} \backslash \bar{B}_{R_{1}}} C L^{2}|\nabla z|^{2} .
$$

It follows from (2.17) that

$$
P \geq \int_{B_{R_{3}} \backslash \bar{B}_{R_{1}}} \frac{1}{2} p^{3} \beta^{2} \Lambda^{-2} r^{-2 p-2}|z|^{2}-\int_{B_{R_{3}} \backslash \bar{B}_{R_{1}}} C L^{2}|\nabla z|^{2},
$$

provided that $p \geq 2 \Lambda^{2} L^{2}$. Since

$$
|Q| \leq \int_{\partial\left(B_{R_{3}} \backslash \bar{B}_{R_{1}}\right)} 2 \Lambda^{2} p^{2} \beta^{2} r^{-2 p-1}|z|^{2},
$$

the conclusion follows.

Using Lemmas 1 and 2, we can prove the following result.

Lemma 3. Let $d \geq 2, \beta \in \mathbb{R}$, and $v \in H^{2}\left(B_{R_{3}} \backslash \bar{B}_{R_{1}}\right)$. There exists a positive constant $p_{\Lambda, L} \geq 1$ such that if $p \geq p_{\Lambda, L}$ and $|\beta| R_{3}^{-p} \geq 2$, then

$$
\begin{aligned}
& \int_{B_{R_{3}} \backslash \bar{B}_{R_{1}}} \frac{r^{p+2} e^{2 \beta r^{-p}}}{2 p|\beta|}[\operatorname{div}(M \nabla v)]^{2}+\int_{B_{R_{3}} \backslash \bar{B}_{R_{1}}} C L^{2} e^{2 \beta r^{-p}}|\nabla v|^{2} \\
&+\int_{\partial\left(B_{R_{3}} \backslash \bar{B}_{R_{1}}\right)} C L^{2} p^{2} \beta^{2} r^{-2 p-1} e^{2 \beta r^{-p}}|v|^{2}+\int_{\partial\left(B_{\left.R_{3} \backslash \bar{B}_{R_{1}}\right)} C L^{2} r e^{2 \beta r^{-p}}|\nabla v|^{2}\right.} \\
& \geq \int_{B_{R_{3}} \backslash \bar{B}_{R_{1}}} \frac{1}{2} \Lambda^{-2} p^{3} \beta^{2} r^{-2 p-2} e^{2 \beta r^{-p}}|v|^{2}
\end{aligned}
$$

for some positive constant $C$ depending only on $d$.

Proof. Set

$$
z=e^{\beta r^{-p}} v \quad \text { equivalently } v=e^{-\beta r^{-p}} z .
$$

Since $\operatorname{div}(M \nabla(g h))=2 \nabla h \cdot M \nabla g+h \operatorname{div}(M \nabla g)+g \operatorname{div}(M \nabla h)(M$ is symmetric $)$, it follows that

$$
\operatorname{div}(M \nabla v)=2 \beta p r^{-p-2} e^{-\beta r^{-p}} x \cdot M \nabla z+e^{-\beta r^{-p}} \operatorname{div}(M \nabla z)+z \operatorname{div}\left(M \nabla e^{-\beta r^{-p}}\right) .
$$

Using the inequality $(a+b+c)^{2} \geq 2 a(b+c)$, we obtain

$$
\begin{aligned}
& \frac{1}{2}[\operatorname{div}(M \nabla v)]^{2} \\
& \quad \geq 2|\beta| p r^{-p-2} e^{-\beta r^{-p}}(x \cdot M \nabla z)\left(e^{-\beta r^{-p}} \operatorname{div}(M \nabla z)+z \operatorname{div}\left(M \nabla e^{-\beta r^{-p}}\right)\right) .
\end{aligned}
$$

This implies

$$
\begin{aligned}
\int_{B_{R_{3}} \backslash \bar{B}_{R_{1}}} \frac{r^{p+2} e^{2 \beta r^{-p}}}{2 p|\beta|}[\operatorname{div}(M \nabla v)]^{2} \geq \int_{B_{R_{3}} \backslash \bar{B}_{R_{1}}} 2(x \cdot M \nabla z) \operatorname{div}(M \nabla z) \\
\quad+\int_{B_{R_{3} \backslash \bar{B}_{R_{1}}} e^{\beta r^{-p}}\left(M x \cdot \nabla|z|^{2}\right) \operatorname{div}\left(M \nabla e^{-\beta r^{-p}}\right) .}
\end{aligned}
$$


Applying Lemmas 1 and 2, we have

$$
\begin{aligned}
\int_{B_{R_{3}} \backslash \bar{B}_{R_{1}}} \frac{r^{p+2} e^{2 \beta r^{-p}}}{2 p|\beta|}[\operatorname{div}(M \nabla v)]^{2} \\
\geq \int_{B_{R_{3}} \backslash \bar{B}_{R_{1}}}\left(\Lambda^{-2} p^{3} \beta^{2} r^{-2 p-2}|z|^{2}-C L^{2}|\nabla z|^{2}\right) \\
\quad-\int_{\partial\left(B_{R_{3}} \backslash \bar{B}_{R_{1}}\right)}\left(C L^{2} p^{2} \beta^{2} r^{-2 p-1}|z|^{2}+C L^{2} r|\nabla z|^{2}\right) .
\end{aligned}
$$

Since $z=e^{\beta r^{-p}} v$,

$$
|\nabla z|^{2} \leq 2 e^{2 \beta r^{-p}}\left(|\nabla v|^{2}+p^{2} \beta^{2} r^{-2 p-2}|v|^{2}\right) .
$$

A combination of (2.19) and (2.20) yields, since $p \geq p_{\Lambda, L}$,

$$
\begin{aligned}
\int_{B_{R_{3}} \backslash \bar{B}_{R_{1}}} & \frac{r^{p+2} e^{2 \beta r^{-p}}}{2 p|\beta|}[\operatorname{div}(M \nabla v)]^{2} \\
\geq & \int_{B_{R_{3}} \backslash \bar{B}_{R_{1}}} e^{2 \beta r^{-p}}\left(\frac{1}{2} \Lambda^{-2} p^{3} \beta^{2} r^{-2 p-2}|v|^{2}-C L^{2}|\nabla v|^{2}\right) \\
& \quad-\int_{\partial\left(B_{R_{3}} \backslash \bar{B}_{R_{1}}\right)} e^{2 \beta r^{-p}}\left(C L^{2} p^{2} \beta^{2} r^{-2 p-1}|v|^{2}+C L^{2} r|\nabla v|^{2}\right) .
\end{aligned}
$$

The conclusion follows.

We also have

Lemma 4. Let $d \geq 2, \beta \in \mathbb{R}$, and $v \in H^{2}\left(B_{R_{3}} \backslash \bar{B}_{R_{1}}\right)$. There exists a positive constant $p_{\Lambda, L} \geq 1$ such that if $p \geq p_{\Lambda, L}$ and $|\beta| R_{3}^{-p} \geq 2$, then

$$
\begin{aligned}
& \int_{B_{R_{3}} \backslash \bar{B}_{R_{1}}} e^{2 \beta r^{-p}} v \operatorname{div}(M \nabla v)+\int_{B_{R_{3}} \backslash \bar{B}_{R_{1}}} e^{2 \beta r^{-p}}|\nabla v|^{2} \\
& \leq \int_{B_{R_{3}} \backslash \bar{B}_{R_{1}}} C \beta^{2} p^{2} r^{-2 p-2} e^{2 \beta r^{-p}}|v|^{2}+\int_{\partial\left(B_{R_{3}} \backslash \bar{B}_{R_{1}}\right)} C e^{2 \beta r^{-p}}\left(r|\nabla v|^{2}+r^{-1}|v|^{2}\right),
\end{aligned}
$$

for some positive constant $C$ depending only on $d, \Lambda$, and $L$.

Proof. We have

$$
\begin{aligned}
& -\int_{B_{R_{3}} \backslash \bar{B}_{R_{1}}} e^{2 \beta r^{-p}} v \operatorname{div}(M \nabla v) \\
& \quad=\int_{B_{R_{3}} \backslash \bar{B}_{R_{1}}} M \nabla v \cdot \nabla\left(e^{2 \beta r^{-p}} v\right)-\int_{\partial\left(B_{R_{3}} \backslash \bar{B}_{R_{1}}\right)} e^{2 \beta r^{-p}} v M \nabla v \cdot \nu .
\end{aligned}
$$

On the other hand,

$$
\begin{aligned}
\int_{B_{R_{3}} \backslash \bar{B}_{R_{1}}} M \nabla v \cdot \nabla\left(e^{2 \beta r^{-p}} v\right) \\
\quad=\int_{B_{R_{3}} \backslash \bar{B}_{R_{1}}}\left(e^{2 \beta r^{-p}} M \nabla v \cdot \nabla v-2 \beta p r^{-p-2} e^{2 \beta r^{-p}} v M \nabla v \cdot x\right)
\end{aligned}
$$


and

$$
\int_{\partial\left(B_{R_{3}} \backslash \bar{B}_{R_{1}}\right)} e^{2 \beta r^{-p}} v M \nabla v \cdot \nu \leq \int_{\partial\left(B_{R_{3}} \backslash \bar{B}_{R_{1}}\right)} e^{2 \beta r^{-p}}\left(r|\nabla v|^{2}+L^{2} r^{-1}|v|^{2}\right) .
$$

Since

$$
2 \beta p r^{-p-2} v M \nabla v \cdot x \leq \frac{1}{2} \Lambda^{-1}|\nabla v|^{2}+8 \beta^{2} p^{2} L^{2} \Lambda r^{-2 p-2}|v|^{2},
$$

we derive from (2.21), (2.22), and (2.23) that

$$
\begin{aligned}
& \int_{B_{R_{3}} \backslash \bar{B}_{R_{1}}} e^{2 \beta r^{-p}} v \operatorname{div}(M \nabla v)+\int_{B_{R_{3}} \backslash \bar{B}_{R_{1}}} \frac{1}{2} \Lambda^{-1} e^{2 \beta r^{-p}}|\nabla v|^{2} \\
& \leq \int_{B_{R_{3}} \backslash \bar{B}_{R_{1}}} C \beta^{2} p^{2} r^{-2 p-2} e^{2 \beta r^{-p}}|v|^{2}+\int_{\partial\left(B_{R_{3}} \backslash \bar{B}_{R_{1}}\right)} C e^{2 \beta r^{-p}}\left(r|\nabla v|^{2}+r^{-1}|v|^{2}\right) .
\end{aligned}
$$

The conclusion follows.

Combining the inequalities in Lemmas 3 and 4 we obtain

Lemma 5. Let $d \geq 2, \beta \in \mathbb{R}$, and $v \in H^{2}\left(B_{R_{3}} \backslash \bar{B}_{R_{1}}\right)$. There exists a positive constant $p_{\Lambda, L} \geq 1$ such that if $p \geq p_{\Lambda, L}$ and $|\beta| \geq 2 R_{3}^{-p}$, then

$$
\begin{aligned}
\int_{B_{R_{3}} \backslash \bar{B}_{R_{1}}} e^{2 \beta r^{-p}}|\beta| p\left(p^{3} \beta^{2} r^{-2 p-2} e^{2 \beta r^{-p}}|v|^{2}+|\nabla v|^{2}\right) \\
\leq C \int_{B_{R_{3}} \backslash \bar{B}_{R_{1}}} r^{p+2} e^{2 \beta r^{-p}}|\operatorname{div}(M \nabla v)|^{2} \\
\quad+C \int_{\partial\left(B_{R_{3}} \backslash \bar{B}_{R_{1}}\right)}|\beta| p e^{2 \beta r^{-p}}\left(r|\nabla v|^{2}+p^{2} \beta^{2} r^{-2 p-1}|v|^{2}\right),
\end{aligned}
$$

for some positive constant $C$ depending only on $d, \Lambda$, and $L$.

Proof. Note that

$$
|v \operatorname{div}(M \nabla v)| \leq p|\beta|^{2}|v|^{2} r^{-2 p-2}+\frac{4}{p|\beta|}|\operatorname{div}(M \nabla v)|^{2} r^{p+2} .
$$

The conclusion now follows from Lemmas 3 and 4 . The details are left to the reader.

\subsection{Proof of Theorem 2. Let}

$$
1<\lambda<\lambda_{0}
$$

(which will be defined later) and set

$$
D=B_{\lambda R_{3}} \backslash \bar{B}_{R_{1} / \lambda} .
$$

Let $u_{1} \in H^{1}\left(D \backslash \partial B_{R_{1}}\right)$ and $u_{3} \in H^{1}\left(D \backslash \partial B_{R_{3}}\right)$ be respectively the unique solution to

$$
\left\{\begin{array}{cl}
\operatorname{div}\left(M \nabla u_{1}\right)=0 & \text { in } D \backslash \partial B_{R_{1}}, \\
{\left[u_{1}\right]=v ;\left[M \nabla u_{1} \cdot \nu\right]=M \nabla v \cdot \nu} & \text { on } \partial B_{R_{1}}, \\
u_{1}=0 & \text { on } \partial D,
\end{array}\right.
$$


and

$$
\left\{\begin{array}{cl}
\operatorname{div}\left(M \nabla u_{3}\right)=0 & \text { in } D \backslash B_{R_{3}}, \\
{\left[u_{3}\right]=v ;\left[M \nabla u_{3} \cdot \nu\right]=M \nabla v \cdot \nu} & \text { on } \partial B_{R_{3}}, \\
u_{3}=0 & \text { on } \partial D .
\end{array}\right.
$$

Here and in what follows, [.] denotes the jump across a sphere and $\nu$ denotes the unit outward normal vector on a sphere. It follows that

$$
\left\|u_{1}\right\|_{H^{1}\left(D \backslash \partial B_{R_{1}}\right)} \leq C\|v\|_{\mathbf{H}\left(\partial B_{R_{1}}\right)}, \quad\left\|u_{1}\right\|_{H^{3 / 2}\left(\partial B_{R_{1} / \gamma}\right)} \leq C\|v\|_{\mathbf{H}\left(\partial B_{R_{1}}\right)}
$$

and

$$
\left\|u_{3}\right\|_{H^{1}\left(D \backslash \partial B_{R_{3}}\right)} \leq C\|v\|_{\mathbf{H}\left(\partial B_{R_{3}}\right)}, \quad\left\|u_{3}\right\|_{H^{3 / 2}\left(\partial B_{\gamma R_{3}}\right)} \leq C\|v\|_{\mathbf{H}\left(\partial B_{R_{3}}\right)} .
$$

Here and in what follows in this proof, $C$ denotes a positive constant depending only on the elliptic and the Lipschitz constant of $M, c_{1}, c_{2}, \lambda_{0}, R_{*}, R^{*}$, and $d$. Set

$$
d_{1}=(\lambda-1) R_{1} \quad \text { and } \quad d_{3}=(\lambda-1) R_{3} / \lambda .
$$

Let $\varphi_{1}, \varphi_{3} \in C_{c}^{2}\left(\mathbb{R}^{d}\right)$ be such that

$$
\varphi_{1}=\left\{\begin{array}{l}
1 \quad \text { in } B_{R_{1}+d_{1} / 3} \backslash B_{R_{1}}, \\
0 \quad \text { in } \mathbb{R}^{d} \backslash\left(B_{R_{1}+d_{1} / 2} \backslash B_{R_{1} / \lambda}\right),
\end{array}\right.
$$

and

$$
\varphi_{3}= \begin{cases}1 & \text { in } B_{R_{3}} \backslash B_{R_{3}-d_{3} / 3}, \\ 0 & \text { in } \mathbb{R}^{d} \backslash\left(B_{\lambda R_{3}} \backslash B_{R_{3}-d_{3} / 2}\right) .\end{cases}
$$

Define

$$
V=\left\{\begin{array}{cl}
v-\varphi_{1} u_{1}-\varphi_{3} u_{3} & \text { in } B_{R_{3}} \backslash \bar{B}_{R_{1}}, \\
-\varphi_{1} u_{1}-\varphi_{3} u_{3} & \text { in } D \backslash\left(B_{R_{3}} \backslash \bar{B}_{R_{1}}\right) .
\end{array}\right.
$$

Applying Lemma 5, we obtain, for $|\beta|>2\left(\gamma R_{3}\right)^{p}$,

$$
\begin{aligned}
& C \int_{D} e^{2 \beta r^{-p}} \beta\left(\beta^{2}|V|^{2}+|\nabla V|^{2}\right) \\
& \quad \leq \int_{D} e^{2 \beta r^{-p}}|\operatorname{div}(M \nabla V)|^{2}+\int_{\partial D}|\beta| e^{2 \beta r^{-p}}\left(|\nabla V|^{2}+\beta^{2}|V|^{2}\right) .
\end{aligned}
$$

The proof is now quite standard and divided into two cases.

Case 1. $\|v\|_{\mathbf{H}\left(\partial B_{R_{1}}\right)} \leq\|v\|_{\mathbf{H}\left(\partial B_{R_{3}}\right)}$. We deduce from (2.28) that for $\beta \geq \beta_{0}:=$ $\max \left\{1,2\left(\gamma_{0} R^{*}\right)^{p}\right\}$

$$
C \int_{D} e^{2 \beta r^{-p}}\left(|V|^{2}+|\nabla V|^{2}\right) \leq \beta^{2} e^{2 \beta \hat{R}_{3}^{-p}}\|v\|_{\mathbf{H}\left(\partial B_{R_{3}}\right)}^{2}+\beta^{2} e^{2 \beta \hat{R}_{1}^{-p}}\|v\|_{\mathbf{H}\left(\partial B_{R_{1}}\right)}^{2}
$$

where

$$
\hat{R}_{3}=R_{3} / \lambda \quad \text { and } \quad \hat{R}_{1}=R_{1} / \lambda
$$

This implies

$$
C\|v\|_{\mathbf{H}\left(\partial B_{R_{2}}\right)} \leq \beta e^{\beta\left(\hat{R}_{3}^{-p}-R_{2}^{-p}\right)}\|v\|_{\mathbf{H}\left(\partial B_{R_{3}}\right)}+\beta e^{\beta\left(\hat{R}_{1}^{-p}-R_{2}^{-p}\right)}\|v\|_{\mathbf{H}\left(\partial B_{R_{1}}\right)} .
$$


Define $\alpha^{\prime} \in(0,1)$ and $\beta>0$ as follows:

$\alpha^{\prime}=\frac{R_{2}^{-p}-\hat{R}_{3}^{-p}}{\hat{R}_{1}^{-p}-\hat{R}_{3}^{-p}} \quad$ and $\quad \beta\left(R_{2}^{-p}-\hat{R}_{1}^{-p}\right)=\left(1-\alpha^{\prime}\right) \ln \left(\|v\|_{\mathbf{H}\left(\partial B_{R_{3}}\right)} /\|v\|_{\mathbf{H}\left(\partial B_{R_{1}}\right)}\right)$ 3

Note that $0<\alpha^{\prime}<1$ since $R_{2}<R_{3} / \gamma$. We assume that $\|v\|_{\mathbf{H}\left(\partial B_{R_{3}}\right)}>C\|v\|_{\mathbf{H}\left(\partial B_{R_{1}}\right)}$ for some large $C$ such that $\beta \geq \max \left\{2 R_{3}^{-p}, 2, \beta_{0}\right\}$ since if $\|v\|_{\mathbf{H}\left(\partial B_{R_{3}}\right)}<C\|v\|_{\mathbf{H}\left(\partial B_{R_{1}}\right)}$, the conclusion holds for any $\alpha \in(0,1)$ by taking $\beta=\max \left\{2 R_{3}^{-p}, 2, \beta_{0}\right\}$ in (2.30). It follows from (2.30) and the choice of $\alpha^{\prime}$ and $\beta$ that

$$
\|v\|_{\mathbf{H}\left(\partial B_{R_{2}}\right)} \leq C \beta\|v\|_{\mathbf{H}\left(\partial B_{R_{1}}\right)}^{\alpha^{\prime}}\|v\|_{\mathbf{H}\left(\partial B_{R_{3}}\right)}^{1-\alpha^{\prime}} .
$$

Define

$$
\alpha:=\frac{R_{2}^{-2 p}-R_{3}^{-2 p}}{R_{1}^{-2 p}-R_{3}^{-2 p}} .
$$

It is clear that $\alpha<\frac{R_{2}^{-p}-R_{3}^{-p}}{R_{1}^{-p}-R_{3}^{-p}}$. Hence, by choosing $\lambda$ close to 1 ,

$$
\alpha<\alpha^{\prime} \quad \forall R_{2} \in\left(\gamma_{0} R_{1}, R_{3} / \gamma_{0}\right) .
$$

A combination of (2.31) and (2.33) implies

$$
\|v\|_{\mathbf{H}\left(\partial B_{R_{2}}\right)} \leq C\|v\|_{\mathbf{H}\left(\partial B_{R_{1}}\right)}^{\alpha}\|v\|_{H\left(\partial B_{R_{3}}\right)}^{1-\alpha} .
$$

Case 2. $\|v\|_{\mathbf{H}\left(\partial B_{R_{1}}\right)} \geq\|v\|_{\mathbf{H}\left(\partial B_{R_{3}}\right)}$. The proof is similar to the previous case by considering $\beta<-2\left(\gamma R_{3}\right)^{-p}$. The details are left to the reader.

\section{Cloaking using complementary media. Proof of Theorem 1}

This section containing two subsections is devoted to the proof of Theorem 1 , In the first subsection, we present two useful lemmas. The proof of Theorem 1 is given in the second subsection.

3.1. Preliminaries. In this section, we present two lemmas which will be used in the proof of Theorems 1 and 3 . The first lemma is on a change of variables and follows from [18, Lemma 1].

Lemma 6. Let $d \geq 2, k>0$, and $0<R_{1}<R_{2}<R_{3}$ with $R_{3}=R_{2}^{2} / R_{1}$. Let $a \in\left[L^{\infty}\left(B_{R_{3}} \backslash \bar{B}_{R_{2}}\right)\right]^{d \times d}$ be a matrix-valued function, $\sigma \in L^{\infty}\left(B_{R_{3}} \backslash \bar{B}_{R_{2}}\right)$ a complex function, and $K: B_{R_{2}} \backslash \bar{B}_{R_{1}} \rightarrow B_{R_{3}} \backslash \bar{B}_{R_{2}}$ the Kelvin transform with respect to $\partial B_{R_{2}}$, i.e.,

$$
K(x)=R_{2}^{2} x /|x|^{2} .
$$

For $v \in H^{1}\left(B_{R_{3}} \backslash \bar{B}_{R_{2}}\right)$, define $w=v \circ K^{-1}$. Then

$$
\operatorname{div}(a \nabla v)+k^{2} \sigma v=0 \text { in } B_{R_{3}} \backslash \bar{B}_{R_{2}}
$$

if and only if

$$
\operatorname{div}\left(K_{*} a \nabla w\right)+k^{2} K_{*} \sigma w=0 \text { in } B_{R_{2}} \backslash \bar{B}_{R_{1}} .
$$

Moreover,

$$
w=v \quad \text { and } \quad K_{*} a \nabla w \cdot \nu=-a \nabla v \cdot \nu \text { on } \partial B_{R_{2}} .
$$

The second lemma is a stability estimate for solutions of (1.10).

\footnotetext{
${ }^{3}$ Here we assume that $\|v\|_{\mathbf{H}\left(\partial B_{R_{1}}\right)} \neq 0$ since otherwise $v=0$. This fact is a consequence of the unique continuation principle and can be obtained from 2.30 by letting $\beta \rightarrow \infty$.
} 
Lemma 7. Let $0<\delta<1$ and $f \in L^{2}(\Omega)$, and let $A \in\left[L^{\infty}(\Omega)\right]^{d \times d}$ and $\Sigma \in$ $L^{\infty}(\Omega, \mathbb{C})$ be such that $A$ is Lipschitz and uniformly elliptic, $\Im(\Sigma) \geq 0$, and $\Re(\Sigma) \geq$ $\lambda>0$ for some $\lambda$. There exists a unique solution $u_{\delta} \in H_{0}^{1}(\Omega)$ of (1.10). Moreover,

$$
\left\|u_{\delta}\right\|_{H^{1}(\Omega)}^{2} \leq C\left(\delta^{-1}\|f\|_{L^{2}(\Omega)}\left\|u_{\delta}\right\|_{L^{2}(\operatorname{supp} f)}+\|f\|_{L^{2}(\Omega)}^{2}\right)
$$

for some positive constant $C$ independent of $\delta$ and $f$.

Lemma 7 is a variant of [18, Lemma 1]. The case $k=0$ and its variant in the case $k>0$ were considered in [21] and [19], respectively. The proof is similar to the one of [18, Lemma 1]. For the convenience of the reader, we present the proof.

Proof. The existence and uniqueness of $u_{\delta}$ are given in [18. We only establish (3.1) by contradiction. Assume that (3.1) is not true. Then there exist $\delta_{n} \rightarrow 0$ and $\left(f_{n}\right) \subset L^{2}(\Omega)$ such that

$$
\left\|u_{n}\right\|_{H^{1}(\Omega)}=1 \text { and } \frac{1}{\delta_{n}}\left\|f_{n}\right\|_{L^{2}(\Omega)}\left\|u_{n}\right\|_{L^{2}\left(\operatorname{supp} f_{n}\right)}+\left\|f_{n}\right\|_{L^{2}(\Omega)}^{2} \rightarrow 0
$$

as $n \rightarrow \infty$, where $u_{n} \in H_{0}^{1}(\Omega)$ is the unique solution to

$$
\operatorname{div}\left(s_{\delta_{n}} A \nabla u_{n}\right)+k^{2} s_{0} \Sigma u_{n}=f_{n} \text { in } \Omega .
$$

Without loss of generality, one may assume that $u_{n} \rightarrow u$ weakly in $H^{1}(\Omega)$ and strongly in $L^{2}(\Omega)$; moreover, $u \in H_{0}^{1}(\Omega)$ and $u$ satisfies

$$
\operatorname{div}\left(s_{0} A \nabla u\right)+k^{2} s_{0} \Sigma u=0 \text { in } \Omega .
$$

Multiplying equation (3.3) by $\bar{u}_{n}$ (the conjugate of $u_{n}$ ) and integrating on $\Omega$, we have

$$
\int_{\Omega} s_{\delta_{n}} A \nabla u_{n} \cdot \nabla \bar{u}_{n} d x-\int_{\Omega} k^{2} s_{0} \Sigma\left|u_{n}\right|^{2} d x=-\int_{\Omega} f_{n} \bar{u}_{n} d x .
$$

Considering the imaginary part and using the fact that

$$
\frac{1}{\delta_{n}}\left|\int_{\Omega} f_{n} \bar{u}_{n}\right| \leq \frac{1}{\delta}\left\|f_{n}\right\|_{L^{2}(\Omega)}\left\|u_{n}\right\|_{L^{2}\left(\operatorname{supp} f_{n}\right)} \rightarrow 0 \text { as } n \rightarrow \infty \text { by (3.2), }
$$

we obtain, by (1.6),

$$
\left\|\nabla u_{n}\right\|_{L^{2}\left(B_{r_{2}} \backslash \bar{B}_{r_{1}}\right)} \rightarrow 0 \text { as } n \rightarrow \infty .
$$

Since $\operatorname{div}\left(A \nabla u_{n}\right)+k^{2} \Sigma u_{n}=f_{n}$ in $B_{r_{2}} \backslash B_{r_{1}}$ and $f_{n} \rightarrow 0$ in $L^{2}(\Omega)$, it follows from (3.5) that $u_{n} \rightarrow 0$ in the distributional sense. This in turn implies

$$
\left\|u_{n}\right\|_{L^{2}\left(B_{r_{2}} \backslash B_{r_{1}}\right)} \rightarrow 0 \text { as } n \rightarrow \infty .
$$

A combination of (3.5) and (3.6) yields

$$
\left\|u_{n}\right\|_{H^{1}\left(B_{r_{2}} \backslash B_{r_{1}}\right)} \rightarrow 0 \text { as } n \rightarrow \infty .
$$

Hence

$$
u=0 \text { in } B_{r_{2}} \backslash B_{r_{1}}
$$

and

$$
\begin{aligned}
\left\|u_{n}\right\|_{H^{1 / 2}\left(\partial B_{r_{2}}\right)} & +\left\|u_{n}\right\|_{H^{1 / 2}\left(\partial B_{r_{1}}\right)}+\left\|A \nabla u_{n} \cdot \nu\right\|_{H^{-1 / 2}\left(\partial B_{r_{2}}\right)} \\
& +\left\|A \nabla u_{n} \cdot \nu\right\|_{H^{-1 / 2}\left(\partial B_{r_{1}}\right)} \rightarrow 0 \text { as } n \rightarrow \infty .
\end{aligned}
$$

Since $u=0$ in $B_{r_{2}} \backslash B_{r_{1}}$ and $u$ satisfies (3.4), it follows from the unique continuation principle that $u=0$ in $\Omega$. Hence, since $u_{n} \rightarrow u$ in $L^{2}(\Omega)$,

$$
u_{n} \rightarrow 0 \text { in } L^{2}(\Omega) \text { as } n \rightarrow \infty \text {. }
$$


Multiplying (3.3) by $\bar{u}_{n}$ and integrating on $\Omega \backslash B_{r_{2}}$, we have

$\int_{\Omega \backslash B_{r_{2}}} A \nabla u_{n} \cdot \nabla \bar{u}_{n} d x-\int_{\Omega \backslash B_{r_{2}}} k^{2} s_{0} \Sigma\left|u_{n}\right|^{2} d x=-\int_{\Omega} f_{n} \bar{u}_{n} d x+\int_{\partial B_{r_{2}}} A \nabla u_{n} \cdot \nu \bar{u}_{n}$.

Using (3.8) and (3.9), we obtain

$$
\left\|\nabla u_{n}\right\|_{L^{2}\left(\Omega \backslash B_{r_{2}}\right)} \rightarrow 0 \text { as } n \rightarrow \infty .
$$

Similarly,

$$
\left\|\nabla u_{n}\right\|_{L^{2}\left(B_{r_{1}}\right)} \rightarrow 0 \text { as } n \rightarrow \infty .
$$

Combining (3.7), (3.9), (3.10), and (3.11), we obtain

$$
\left\|u_{n}\right\|_{H^{1}(\Omega)} \rightarrow 0 \text { as } n \rightarrow \infty
$$

which contradicts (3.2). The proof is complete.

3.2. Proof of Theorem 1, We use the approach in [21] with some modifications from [19] so that the same proof also gives the result on illusion optics (Theorem 3 in Section 4). However, instead of applying the standard three sphere inequality as in 21, we use Theorem 2

We have, by Lemma 7 .

$$
\left\|u_{\delta}\right\|_{H^{1}(\Omega)}^{2} \leq C\left(\delta^{-1}\|f\|_{L^{2}(\Omega)}\left\|u_{\delta}\right\|_{L^{2}\left(\Omega \backslash B_{r_{3}}\right)}+\|f\|_{L^{2}(\Omega)}^{2}\right) .
$$

As in [21], let $u_{1, \delta}$ be the reflection of $u_{\delta}$ through $\partial B_{r_{2}}$ by $F$, i.e.,

$$
u_{1, \delta}=u_{\delta} \circ F^{-1} \text { in } \mathbb{R}^{d} \backslash \bar{B}_{r_{2}},
$$

and let $u_{2, \delta}$ be the reflection of $u_{1, \delta}$ through $\partial B_{r_{2}}$ by $G$, i.e.,

$$
u_{2, \delta}=u_{1, \delta} \circ G^{-1} \text { in } B_{r_{3}} .
$$

By Lemma 6,

$$
\begin{gathered}
\operatorname{div}\left(A \nabla u_{1, \delta}\right)+\frac{1}{1-i \delta} k^{2} \Sigma u_{1, \delta}=0 \text { in } B_{r_{3}} \backslash B_{r_{2}}, \\
\Delta u_{2, \delta}+k^{2} u_{2, \delta}=0 \text { in } B_{r_{3}} .
\end{gathered}
$$

Applying Lemma 6 again and using the fact that $F_{*} A=A$ in $B_{r_{3}} \backslash B_{r_{2}}$, we have

$$
u_{1, \delta}=\left.u_{\delta}\right|_{+} \text {on } \partial B_{r_{2}} \quad \text { and } \quad(1-i \delta) A \nabla u_{1, \delta} \cdot \nu=\left.A \nabla u_{\delta} \cdot \nu\right|_{+} \text {on } \partial B_{r_{2}} .
$$

Let $V_{1, \delta} \in H^{1}\left(B_{r_{3}} \backslash B_{r_{2}}\right)$ be the unique solution to

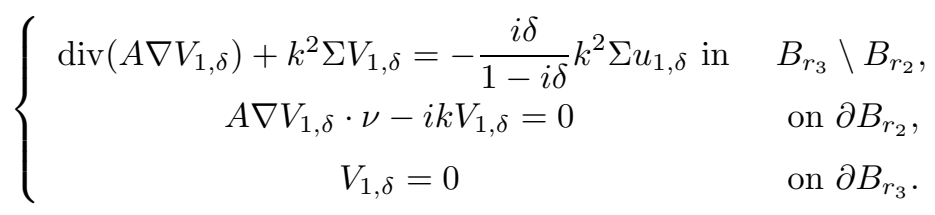

By Fredholm's theory,

$$
\left\|V_{1, \delta}\right\|_{H^{1}\left(B_{r_{3}} \backslash B_{r_{2}}\right)} \leq C \delta\left\|u_{\delta}\right\|_{H^{1}(\Omega)} .
$$

Define $U_{1, \delta}$ in $B_{r_{3}} \backslash B_{r_{2}}$ as

$$
U_{1, \delta}=u_{\delta}-u_{1, \delta}-V_{1, \delta}
$$


Then $U_{1, \delta} \in H^{1}\left(B_{r_{3}} \backslash B_{r_{2}}\right)$ and $U_{1, \delta}$ satisfies

$$
\begin{gathered}
\operatorname{div}\left(A \nabla U_{1, \delta}\right)+k^{2} \Sigma U_{1, \delta}=0 \text { in } B_{r_{3}} \backslash B_{r_{2}}, \\
\left\|U_{1, \delta}\right\|_{H^{1 / 2}\left(\partial B_{r_{2}}\right)}+\left\|A \nabla U_{1, \delta} \cdot \nu\right\|_{H^{1 / 2}\left(\partial B_{r_{2}}\right)} \leq C \delta\left\|u_{\delta}\right\|_{H^{1}(\Omega)},
\end{gathered}
$$

and

$$
\left\|U_{1, \delta}\right\|_{H^{1 / 2}\left(\partial B_{r_{3}}\right)}+\left\|A \nabla U_{1, \delta} \cdot \nu\right\|_{H^{1 / 2}\left(\partial B_{r_{3}}\right)} \leq C\left\|u_{\delta}\right\|_{H^{1}(\Omega)} .
$$

Applying Theorem 2, we have

$$
\left\|U_{1, \delta}\right\|_{H^{1 / 2}\left(\partial B_{\gamma r_{2}}\right)}+\left\|A \nabla U_{1, \delta} \cdot \nu\right\|_{H^{1 / 2}\left(\partial B_{\gamma r_{2}}\right)} \leq C \delta^{\alpha}\left\|u_{\delta}\right\|_{H^{1}(\Omega)},
$$

where $\alpha$ is given in (2.8) with $R_{1}=r_{2}, R_{2}=\gamma r_{2}, R_{3}=r_{3}$. By choosing $\gamma_{0}$ close enough to 1 , from (2.8), we can assume that

$$
\alpha>1 / 2 \text {. }
$$

Here is the place where the condition $\gamma<\gamma_{0}$ is required. A combination of (3.19) and (3.21) yields

(3.23) $\left\|u_{\delta}-u_{1, \delta}\right\|_{H^{1 / 2}\left(\partial B_{\gamma r_{2}}\right)}+\left\|A \nabla\left(u_{\delta}-u_{1, \delta}\right) \cdot \nu\right\|_{H^{-1 / 2}\left(\partial B_{\gamma r_{2}}\right)} \leq C \delta^{\alpha}\left\|u_{\delta}\right\|_{H^{1}(\Omega)}$.

In what follows, we assume that $k=1$ for notational ease. Define $U_{2, \delta}$ in $B_{r_{3}} \backslash B_{\gamma r_{2}}$ as

$$
U_{2, \delta}=u_{1, \delta}-u_{2, \delta}+V_{1, \delta}
$$

Then

$$
\Delta U_{2, \delta}+U_{2, \delta}=0 \quad \text { in } B_{r_{3}} \backslash B_{\gamma r_{2}}
$$

and

$$
U_{2, \delta}=0 \quad \text { and } \quad \partial_{r} U_{2, \delta}=-\frac{i \delta}{1-i \delta} \partial_{r} u_{1, \delta}+\partial_{r} V_{1, \delta} \quad \text { on } \partial B_{r_{3}} .
$$

Case 1. $d=2$. As in [19], define

$$
\hat{J}_{n}(t)=2^{n} n ! J_{n}(t) \quad \text { and } \quad \hat{Y}_{n}(t)=-\frac{\pi}{2^{n}(n-1) !} Y_{n}(t),
$$

where $J_{n}$ and $Y_{n}$ are the Bessel and Neumann functions of order $n$. It follows from [5. (3.80) and (3.81)] that

$$
\hat{J}_{n}(t)=t^{n}[1+o(1)]
$$

and

$$
\hat{Y}_{n}(t)=t^{-n}[1+o(1)]
$$

as $n \rightarrow+\infty$.

From (3.24) one can represent $U_{2, \delta}$ as

$$
\begin{aligned}
U_{2, \delta}= & a_{0} \hat{J}_{0}(|x|)+b_{0} \hat{Y}_{0}(|x|) \\
& +\sum_{n=1}^{\infty} \sum_{ \pm}\left[a_{n, \pm} \hat{J}_{n}(|x|)+b_{n, \pm} \hat{Y}_{n}(|x|)\right] e^{ \pm i n \theta} \quad \text { in } B_{r_{3}} \backslash B_{\gamma r_{2}}
\end{aligned}
$$

for $a_{0}, b_{0}, a_{n, \pm}, b_{n, \pm} \in \mathbb{C}(n \geq 1)$. Assume that

$$
\partial_{r} U_{2, \delta}=c_{0}+\sum_{n \geq 1} \sum_{ \pm} c_{n, \pm} e^{ \pm i n \theta} \quad \text { on } \partial B_{r_{3}} .
$$


Then, by (3.18), (3.19), and (3.25),

$$
\left|c_{0}\right|^{2}+\sum_{n \geq 1} \sum_{ \pm} n^{-1}\left|c_{n, \pm}\right|^{2} \sim\left\|\partial_{r} U_{2, \delta}\right\|_{H^{-1 / 2}\left(\partial B_{r_{3}}\right)}^{2} \leq C \delta^{2}\|u\|_{H^{1}(\Omega)}^{2} .
$$

Using (3.25) again, we have

$$
\left\{\begin{array}{c}
a_{n, \pm} \hat{J}_{n}\left(r_{3}\right)+b_{n, \pm} \hat{Y}_{n}\left(r_{3}\right)=0, \\
a_{n, \pm} \hat{J}_{n}^{\prime}\left(r_{3}\right)+b_{n, \pm} \hat{Y}_{n}^{\prime}\left(r_{3}\right)=c_{n, \pm},
\end{array} \quad \text { for } n \geq 0 .\right.
$$

Here we denote $a_{0, \pm}=a_{0} / 2, b_{0, \pm}=b_{0} / 2$, and $c_{0, \pm}=c_{0} / 2$. It follows that

$$
\left\{\begin{array}{l}
a_{n, \pm}=c_{n, \pm} A C_{n}, \\
b_{n, \pm}=c_{n, \pm} B C_{n},
\end{array} \quad \text { for } n \geq 0,\right.
$$

where

$$
A C_{n}=-\frac{\hat{Y}_{n}}{\hat{J}_{n} \hat{Y}_{n}^{\prime}-\hat{J}_{n}^{\prime} \hat{Y}_{n}}\left(r_{3}\right) \quad \text { and } \quad B C_{n}=-\frac{\hat{J}_{n}}{\hat{Y}_{n} \hat{J}_{n}^{\prime}-\hat{Y}_{n}^{\prime} \hat{J}_{n}}\left(r_{3}\right) .
$$

Using (3.26) and (3.27), we derive that

$$
A C_{n}=-\frac{1}{2 n} r_{3}^{1-n}(1+o(1)) \quad \text { and } B C_{n}=\frac{1}{2 n} r_{3}^{1+n}(1+o(1)) .
$$

We now make use of the removing of localized singularity technique introduced in 19,21. Set

$$
\hat{u}_{\delta}(x)=\sum_{n=1}^{\infty} \sum_{ \pm} b_{n, \pm} \hat{Y}_{n}(|x|) e^{ \pm i n \theta} \quad \text { in } B_{r_{3}} \backslash B_{\gamma r_{2}} .
$$

We claim that, for $\gamma r_{2} \leq r \leq r_{3}$,

$$
\left\|U_{2, \delta}-\hat{u}_{\delta}\right\|_{H^{1 / 2}\left(\partial B_{r}\right)}+\left\|\partial_{r} U_{2, \delta}-\partial_{r} \hat{u}_{\delta}\right\|_{H^{-1 / 2}\left(\partial B_{r}\right)} \leq C \delta\left\|u_{\delta}\right\|_{H^{1}(\Omega)} .
$$

Indeed, for $\gamma r_{2} \leq r \leq r_{3}$,

$$
\begin{aligned}
\left\|U_{2, \delta}-\hat{u}_{\delta}\right\|_{H^{1 / 2}\left(\partial B_{r}\right)}^{2} & =\left\|\sum_{n \geq 0} \sum_{ \pm} a_{n, \pm} \hat{J}_{n}(|x|) e^{i n \theta}\right\|_{H^{1 / 2}\left(\partial B_{r}\right)}^{2} \\
& \sim \sum_{n \geq 0} \sum_{ \pm}(n+1)\left|a_{n, \pm}\right|^{2}\left|\hat{J}_{n}(|x|)\right|^{2} \\
& \sim \sum_{n \geq 0} \sum_{ \pm}(n+1)\left|c_{n, \pm} A C_{n}\right|^{2}\left|\hat{J}_{n}(|x|)\right|^{2} \\
& \leq C \sum_{n \geq 0} \sum_{ \pm}(n+1)^{-1}\left|c_{n, \pm}\right|^{2}\left(r / r_{3}\right)^{2 n} .
\end{aligned}
$$

It follows from (3.29) that

$$
\left\|U_{2, \delta}-\hat{u}_{\delta}\right\|_{H^{1 / 2}\left(\partial B_{r}\right)} \leq C \delta\left\|u_{\delta}\right\|_{H^{1}(\Omega)},
$$

for $\gamma r_{2} \leq r \leq r_{3}$. Similarly,

$$
\left\|\partial_{r} U_{2, \delta}-\partial_{r} \hat{u}_{\delta}\right\|_{H^{-1 / 2}\left(\partial B_{r}\right)} \leq C \delta\left\|u_{\delta}\right\|_{H^{1}(\Omega)},
$$


for $\gamma r_{2} \leq r \leq r_{3}$. As a consequence of (3.19) and (3.31), we obtain for $\gamma r_{2} \leq r \leq r_{3}$, $(3.32)$

$$
\left\|u_{1, \delta}-u_{2, \delta}-\hat{u}_{\delta}\right\|_{H^{1 / 2}\left(\partial B_{r}\right)}+\left\|\partial_{r} u_{1, \delta}-\partial u_{2, \delta}-\partial_{r} \hat{u}_{\delta}\right\|_{H^{-1 / 2}\left(\partial B_{r}\right)} \leq C \delta\left\|u_{\delta}\right\|_{H^{1}(\Omega)} .
$$

Define

$$
U_{\delta}=\left\{\begin{array}{cl}
u_{\delta} & \text { in } \Omega \backslash B_{r_{3}} \\
u_{\delta}-\hat{u}_{\delta} & \text { if } x \in B_{r_{3}} \backslash B_{\gamma r_{2}} \\
u_{2, \delta} & \text { if } x \in B_{\gamma r_{2}} .
\end{array}\right.
$$

We have

$$
\operatorname{div}\left(A \nabla U_{\delta}\right)+k^{2} \Sigma U_{\delta}=f \text { in } \Omega \backslash\left(\partial B_{r_{3}} \cup \partial B_{\gamma r_{2}}\right) .
$$

On the other hand, from (3.23) and (3.32), we obtain

$$
\left\|\left[U_{\delta}\right]\right\|_{H^{1 / 2}\left(\partial B_{\gamma r_{2}}\right)}+\left\|\left[\partial_{r} U_{\delta} \cdot \nu\right]\right\|_{H^{-1 / 2}\left(\partial B_{\gamma r_{2}}\right)} \leq C \delta^{\alpha}\left\|u_{\delta}\right\|_{H^{1}(\Omega)}
$$

and

$$
\left\|\left[U_{\delta}\right]\right\|_{H^{1 / 2}\left(\partial B_{r_{3}}\right)}+\left\|\left[\partial_{r} U_{\delta} \cdot \nu\right]\right\|_{H^{-1 / 2}\left(\partial B_{r_{3}}\right)} \leq C \delta^{\alpha}\left\|u_{\delta}\right\|_{H^{1}(\Omega)} .
$$

Using (3.12), we derive that

$$
\begin{aligned}
& \left\|U_{\delta}\right\|_{H^{1}\left(\Omega \backslash\left(\partial B_{r_{3}} \cup \partial B_{\gamma r_{2}}\right)\right)} \\
& \quad \leq C \delta^{\alpha}\left(\delta^{-1 / 2}\left\|U_{\delta}\right\|_{L^{2}\left(\Omega \backslash B_{r_{3}}\right)}^{1 / 2}\|f\|_{L^{2}(\Omega)}^{1 / 2}+\|f\|_{L^{2}(\Omega)}\right)+C\|f\|_{L^{2}(\Omega)} .
\end{aligned}
$$

Since $\alpha>1 / 2$, it follows that $U_{\delta}$ is bounded in $H^{1}\left(\Omega \backslash\left(\partial B_{r_{3}} \cup \partial B_{\gamma r_{2}}\right)\right)$. Without loss of generality, one may assume that $U_{\delta} \rightarrow U$ weakly in $H^{1}\left(\Omega \backslash\left(\partial B_{r_{3}} \cup \partial B_{\gamma r_{2}}\right)\right)$ as $\delta \rightarrow 0$; moreover, $U \in H^{1}(\Omega)$ and

$$
\Delta U+k^{2} U=f \text { in } \Omega \text { and } U=0 \text { on } \partial \Omega .
$$

Hence $U=u$. Since the limit is unique, we have the convergence for the family $\left(U_{\delta}\right)$ as $\delta \rightarrow 0$.

Case 2. $d=3$. Define

$$
\hat{j}_{n}(t)=1 \cdot 3 \cdots(2 n+1) j_{n}(t) \quad \text { and } \quad \hat{y}_{n}=-\frac{y_{n}(t)}{1 \cdot 3 \cdots(2 n-1)},
$$

where $j_{n}$ and $y_{n}$ are the spherical Bessel and Neumann functions of order $n$. Then, for $n$ large enough (see, e.g., [5, (2.38) and (2.39)]),

$$
\hat{j}_{n}(t)=t^{n}(1+O(1 / n)) \quad \text { and } \quad \hat{y}_{n}(r)=t^{-n-1}(1+O(1 / n)) .
$$

Thus one can represent $U_{2, \delta}$ of the form

$$
U_{2, \delta}=\sum_{n=1}^{\infty} \sum_{m=-n}^{n}\left[a_{n}^{m} \hat{j}_{n}(|x|)+b_{n}^{m} \hat{y}_{n}(|x|)\right] Y_{n}^{m}(\hat{x}) \quad \text { in } B_{r_{3}} \backslash B_{r_{0}}
$$

for $a_{m}^{n}, b_{m}^{n} \in \mathbb{C}$ and $\hat{x}=x /|x|$, where $Y_{n}^{m}$ is the spherical function of degree $n$ and of order $m$. The proof now follows similarly as in the case $d=2$. The details are left to the reader. 
Remark 4. Define $V_{\delta}=U_{\delta}-u$ in $\Omega$. Then $V_{\delta} \in H^{1}\left(\Omega \backslash\left(\partial B_{\gamma r_{2}} \cup \partial B_{r_{3}}\right)\right)$,

$$
\Delta V_{\delta}+k^{2} V_{\delta}=0 \text { in } \Omega \backslash\left(\partial B_{\gamma r_{2}} \cup \partial B_{r_{3}}\right), \quad V_{\delta}=0 \text { on } \partial \Omega,
$$

and, from (3.33) and (3.34),

$$
\left\|\left[V_{\delta}\right]\right\|_{H^{1 / 2}\left(\partial B_{\gamma r_{2}} \cup \partial B_{r_{3}}\right)}+\left\|\left[\partial_{r} V_{\delta} \cdot \nu\right]\right\|_{H^{-1 / 2}\left(\partial B_{\gamma r_{2}} \cup \partial B_{r_{3}}\right)} \leq C \delta^{\alpha-1 / 2}\|f\|_{L^{2}(\Omega)} .
$$

It follows that $\left\|V_{\delta}\right\|_{H^{1}\left(\Omega \backslash\left(\partial B_{\gamma r_{2}} \cup \partial B_{r_{3}}\right)\right)} \leq C \delta^{\alpha-1 / 2}\|f\|_{L^{2}}$. This implies that $\left\|u_{\delta}-u\right\|_{H^{1}\left(\Omega \backslash \bar{B}_{r_{3}}\right)} \leq C \delta^{\alpha-1 / 2}\|f\|_{L^{2}}$. Note that $\alpha$ can be close to 1 if $\gamma$ is sufficiently close to 1 (in order to keep the size of the cloaked object unchanged, one needs to have large $r_{2}$; see also Remark 2).

Remark 5. In the proof, we use essentially the fact $(A, \Sigma)=(I, 1)$ in $B_{r_{3}} \backslash B_{\gamma r_{2}}$ to use separation of variables in this region. In fact, this condition is not necessary by using the technique of separation of variables for a general structure in [20].

Remark 6. The construction of the cloak given by (1.4) is not restricted to the Kelvin transforms $F$ (and $G$ ). In fact, one can extend this construction to a general class of reflections considered in [18].

Remark 7 . The condition $\left(F_{*} A, F_{*} \Sigma\right)=(A, \Sigma)$ in $B_{r_{3}} \backslash B_{r_{2}}$ is necessary to ensure that cloaking can be achieved and the localized resonance might take place; see [23] (see also 4] for related results).

Remark 8. Cloaking can also be achieved via schemes generated by changes of variables [7, 14, 31]. Resonance might also appear in this context but for specific frequencies see [9, 16. It was shown in [16] that in the resonance case cloaking might not be achieved and the field inside the cloaked region can depend on the field outside. Cloaking can also be achieved in the time regime via change of variables [26, 27].

\section{ILLUSION OPTICS USING COMPLEMENTARY MEDIA}

We next discuss briefly how to obtain illusion optics in the spirit of Lai et al. in 12 . The scheme used here is a combination of the ones used for cloaking and superlensing in [19,21] and is slightly different from [12]. More precisely, set

$$
m=r_{3}^{2} / r_{2}^{2} \text {. }
$$

Let $a_{c} \in\left[L^{\infty}\left(B_{r_{2} / m}\right)\right]^{d \times d}$ be elliptic and $\sigma_{c} \in L^{\infty}\left(B_{r_{2}^{2} / r_{3}^{2}}, \mathbb{C}\right)$ with $\Im\left(\sigma_{c}\right) \geq 0$. Define

$$
A_{1}, \Sigma_{1}= \begin{cases}A, \Sigma & \text { in } \Omega \backslash B_{r_{2} / m} \\ a_{c}, \sigma_{c} & \text { in } B_{r_{2} / m}\end{cases}
$$

and

$$
\hat{A}_{1}, \hat{\Sigma}_{1}=\left\{\begin{array}{cl}
I, 1 & \text { in } \Omega \backslash B_{r_{2}}, \\
\left(r_{3} / r_{2}\right)^{2-d} a_{c}(x / m),\left(r_{3} / r_{2}\right)^{-d} \sigma_{c}(x / m) & \text { in } B_{r_{2}} .
\end{array}\right.
$$

Recall that $(A, \Sigma)$ is defined in (1.4). We assume that the following equation has only a zero solution in $H_{0}^{1}(\Omega)$ :

$$
\operatorname{div}\left(A_{1} \nabla v\right)+k^{2} \Sigma_{1} v=0 \text { in } \Omega .
$$


We obtain the following result on illusion optics:

Theorem 3. Let $d=2,3$ and $f \in L^{2}(\Omega)$ with $\operatorname{supp} f \subset \Omega \backslash B_{r_{3}}$, and let $u$ and $u_{\delta}$ in $H_{0}^{1}(\Omega)$ be respectively the unique solution of

$$
\operatorname{div}\left(s_{\delta} A_{1} \nabla u_{\delta}\right)+k^{2} s_{0} \Sigma_{1} u_{\delta}=f \quad \text { in } \Omega
$$

and

$$
\operatorname{div}\left(\hat{A}_{1} \nabla u\right)+k^{2} \hat{\Sigma}_{1} u=f \quad \text { in } \Omega .
$$

There exists $\gamma_{0}>1$, depending only on $\Lambda$ and the Lipschitz constant of $\hat{a}$ such that if $1<\gamma<\gamma_{0}$, then

$$
u_{\delta} \rightarrow u \text { weakly in } H^{1}\left(\Omega \backslash B_{r_{3}}\right) \text { as } \delta \rightarrow 0 .
$$

For an observer outside $B_{r_{3}}$, the medium in $B_{r_{3}}$ looks like $\left(\hat{A}_{1}, \hat{\Sigma}_{1}\right)$ : one has illusion optics.

Proof. The proof is similar to the one of Theorem 1. Note that in the proof of Theorem 1 we do not use the information of the medium inside $B_{r_{2} / m}$. The details are left to the reader.

\section{REFERENCES}

[1] S. Agmon, Unicité et convexité dans les problèmes différentiels (French), Séminaire de Mathématiques Supérieures, No. 13 (Été, 1965), Les Presses de l'Université de Montréal, Montreal, Que., 1966. MR0252808 (40 \#6025)

[2] G. Alessandrini, L. Rondi, E. Rosset, and S. Vessella, The stability for the Cauchy problem for elliptic equations, Inverse Problems 25 (2009), no. 12, 123004, 47, DOI 10.1088/02665611/25/12/123004. MR2565570(2010k:35517)

[3] H. Ammari, G. Ciraolo, H. Kang, H. Lee, and G. W. Milton, Spectral theory of a NeumannPoincaré-type operator and analysis of cloaking due to anomalous localized resonance, Arch. Ration. Mech. Anal. 208 (2013), no. 2, 667-692, DOI 10.1007/s00205-012-0605-5. MR.3035988

[4] A. S. Bonnet-Ben Dhia, P. Ciarlet Jr., and C. M. Zwölf, Time harmonic wave diffraction problems in materials with sign-shifting coefficients, J. Comput. Appl. Math. 234 (2010), no. 6, 1912-1919, DOI 10.1016/j.cam.2009.08.041. MR2644187 (2011m:78009)

[5] D. Colton and R. Kress, Inverse acoustic and electromagnetic scattering theory, 3rd ed., Applied Mathematical Sciences, vol. 93, Springer, New York, 2013. MR2986407

[6] N. Garofalo and F.-H. Lin, Unique continuation for elliptic operators: a geometric-variational approach, Comm. Pure Appl. Math. 40 (1987), no. 3, 347-366, DOI 10.1002/cpa.3160400305. MR882069(88j:35046)

[7] A. Greenleaf, M. Lassas, and G. Uhlmann, On nonuniqueness for Calderón's inverse problem, Math. Res. Lett. 10 (2003), no. 5-6, 685-693, DOI 10.4310/MRL.2003.v10.n5.a11. MR2024725 (2005f:35316)

[8] J. Hadamard, Sur les fonction entières, Bull. Soc. Math. France 24 (1896), 94-96.

[9] R. V. Kohn, D. Onofrei, M. S. Vogelius, and M. I. Weinstein, Cloaking via change of variables for the Helmholtz equation, Comm. Pure Appl. Math. 63 (2010), no. 8, 973-1016, DOI 10.1002/cpa.20326. MR2642383 (2011j:78004)

[10] R. V. Kohn, J. Lu, B. Schweizer, and M. I. Weinstein, A variational perspective on cloaking by anomalous localized resonance, Comm. Math. Phys. 328 (2014), no. 1, 1-27, DOI 10.1007/s00220-014-1943-y. MR3196978

[11] Y. Lai, H. Chen, Z. Zhang, and C. T. Chan, Complementary media invisibility cloak that cloaks objects at a distance outside the cloaking shell, Phys. Rev. Lett. 102 (2009), 093901.

[12] Y. Lai and J. Ng and H. Chen and D. Han and J. Xiao and Z. Zhang and C. T. Chan, Illusion optics: The optical transformation of an object into another object, Phys. Rev. Lett. 102 (2009), 253902.

[13] E. M. Landis, Some questions in the qualitative theory of second-order elliptic equations (case of several independent variables) (Russian), Uspehi Mat. Nauk 18 (1963), no. 1 (109), 3-62. MR0150437 (27 \#435) 
[14] U. Leonhardt, Optical conformal mapping, Science 312 (2006), no. 5781, 1777-1780, DOI 10.1126/science.1126493. MR2237569

[15] G. W. Milton and N.-A. P. Nicorovici, On the cloaking effects associated with anomalous localized resonance, Proc. R. Soc. Lond. Ser. A Math. Phys. Eng. Sci. 462 (2006), no. 2074, 3027-3059, DOI 10.1098/rspa.2006.1715. MR 2263683 (2008e:78018)

[16] H.-M. Nguyen, Approximate cloaking for the Helmholtz equation via transformation optics and consequences for perfect cloaking, Comm. Pure Appl. Math. 65 (2012), no. 2, 155-186, DOI 10.1002/cpa.20392. MR2855543

[17] H.-M. Nguyen, Cloaking via anomalous localized resonance. A connection between the localized resonance and the blow up of the power for doubly complementary media (English, with English and French summaries), C. R. Math. Acad. Sci. Paris 353 (2015), no. 1, 41-46, DOI 10.1016/j.crma.2014.10.014. MR.3285145

[18] H.-M. Nguyen, Asymptotic behavior of solutions to the Helmholtz equations with sign changing coefficients, Trans. Amer. Math. Soc. 367 (2015), no. 9, 6581-6595, DOI 10.1090/S00029947-2014-06305-8. MR 3356948

[19] H.-M. Nguyen, Superlensing using complementary media, Ann. Inst. H. Poincaré Anal. Non Linéaire 32 (2015), no. 2, 471-484, DOI 10.1016/j.anihpc.2014.01.004. MR3325246

[20] H.-M. Nguyen, Cloaking via anomalous localized resonance for doubly complementary media in the quasistatic regime, J. Eur. Math. Soc. (JEMS) 17 (2015), no. 6, 1327-1365, DOI 10.4171/JEMS/532. MR3353803

[21] H.-M. Nguyen, Cloaking using complementary media in the quasistatic regime, Ann. Inst. H. Poincaré Anal. Non Linéaire, to appear, DOI 10.1016/j.anihpc.2015.06.004.

[22] H.-M. Nguyen, Reflecting complementary media and superlensing using complementary media for electromagnetic waves, submitted.

[23] H.-M. Nguyen, Limiting absorption principle and well-posedness for the Helmholtz equation with sign changing coefficients, submitted, arXiv.org/abs/1507.01730.

[24] H.-M. Nguyen, Cloaking using complementary media for electromagnetic waves, preprint.

[25] H.-M. Nguyen and L. H. Nguyen, Localized and complete resonance in plasmonic structures, ESAIM Math. Model. Numer. Anal. 49 (2015), no. 3, 741-754, DOI 10.1051/m2an/2014051. MR.3342226

[26] H.-M. Nguyen and M. S. Vogelius, Approximate cloaking for the full wave equation via change of variables, SIAM J. Math. Anal. 44 (2012), no. 3, 1894-1924, DOI 10.1137/110833154. MR2982735

[27] H-M. Nguyen and M. S. Vogelius, Approximate cloaking for the full wave equation: a study of the Lorentz model, submitted.

[28] N. A. Nicorovici, R. C. McPhedran, and G. M. Milton, Optical and dielectric properties of partially resonant composites, Phys. Rev. B 49 (1994), 8479-8482.

[29] J. B. Pendry, Negative refraction makes a perfect lens, Phys. Rev. Lett. 85 (2000), 3966-3969.

[30] J. B. Pendry, Perfect cylindrical lenses, Optics Express 1 (2003), 755-760.

[31] J. B. Pendry, D. Schurig, and D. R. Smith, Controlling electromagnetic fields, Science 312 (2006), no. 5781, 1780-1782, DOI 10.1126/science.1125907. MR2237570

[32] S. A. Ramakrishna and J. B. Pendry, Focusing light using negative refraction, J. Phys.: Condens. Matter 15 (2003), 6345-6364.

[33] S. A. Ramakrishna and J. B. Pendry, Spherical perfect lens: Solutions of Maxwell's equations for spherical geometry, Phys. Rev. B 69 (2004), 115115.

[34] M. H. Protter, Unique continuation for elliptic equations, Trans. Amer. Math. Soc. 95 (1960), 81-91. MR0113030(22 \#3871)

[35] R. A. Shelby, D. R. Smith, and S. Schultz, Experimental verification of a negative index of refraction, Science 292 (2001), 77-79.

[36] V. G. Veselago, The electrodynamics of substances with simultaneously negative values of $\varepsilon$ and $\mu$, Usp. Fiz. Nauk 92 (1964), 517-526.

Mathematics Section, École Polytechnique Fédérale de Lausanne, Station 8, CH1015 Lausanne, Switzerland

E-mail address: hoai-minh.nguyen@epfl.ch

Mathematics Section, École Polytechnique Fédérale de Lausanne, Station 8, CH1015 Lausanne, Switzerland

E-mail address: loc.nguyen@epfl.ch 